\title{
Disposición de excretas en Costa Rica: de los Objetivos de Desarrollo del Milenio a los Objetivos de Desarrollo Sostenible
}

\section{Excreta disposal in Costa Rica: from the Millennial Development Goals to the Sustainable Development Goals}

Darner A. Mora -Alvarado', Carlos Felipe Portuguez-Barquero²

Mora-Alvarado, D; Portuguez-Barquero, C. Disposición de excretas en Costa Rica: de los Objetivos de Desarrollo del Milenio a los Objetivos de Desarrollo Sostenible. Tecnología en Marcha. Diciembre 2019. Vol 32 Especial. Laboratorio Nacional de Aguas. Pág 46-56.

doi) https://doi.org/10.18845/tm.v32i10.4880

Director del Laboratorio Nacional de Aguas. Instituto Costarricense de Acueductos y Alcantarillados. Costa Rica. Correo electróico: dmora@aya.go.cr.

2 Gestor Ambiental. Laboratorio Nacional de Aguas. Instituto Costarricense de Acueductos y Alcantarillados. Costa Rica. Correo electrónico: fportuguez@aya.go.cr. 


\title{
Palabras clave
}

Higiene; saneamiento; tanques sépticos; fuentes de saneamiento administrados de forma segura.

\section{Resumen}

La transición de los Objetivos de Desarrollo del Milenio (ODM) a los Objetivos de Desarrollo Sostenible (ODS) provocó cambios en conceptos clave sobre saneamiento. En este trabajo, se estudió la sustitución de Instalaciones de saneamiento mejoradas por servicios de saneamiento administrados de manera segura. Se analizaron los tanques sépticos utilizando dos escenarios con base en lo establecido por los ODS. Se realizó una comparación entre Costa Rica y el mundo dentro del contexto de saneamiento. La evaluación de los objetivos de eliminación de excretas y saneamiento hasta 2030 para Costa Rica, se realizó utilizando datos de la OMS y del Laboratorio Nacional del Agua (LNA). Los resultados mostraron que, en el escenario donde los tanques sépticos se consideran Servicios de saneamiento administrados de manera segura, el país alcanza $87,6 \%$ de población cubierta. Mientras que cuando no se consideran como Servicios de saneamiento administrados de forma segura, se alcanza un 13\% de cobertura. Costa Rica se ubica en el quinto puesto en cobertura de higiene básica entre 13 países de Latinoamérica. Es necesario un seguimiento estricto de las políticas públicas relacionadas con el saneamiento con el fin mejorar los indicadores del país en el contexto de los ODS.

\section{Keywords}

Hygiene; sanitation; septic tanks; Safely managed sanitation services.

\begin{abstract}
The transition from the Millennium Development Goals (DMG) to the Sustainable Development Goals (SDG) caused changes in key concepts about sanitation. In this work, the replacement of the Improved Sanitation Facilities by the Safely managed sanitation services was studied. Septic tanks were analyzed using two scenarios according to the SDG. A comparison between Costa Rica and the world within the context of sanitation was made. Evaluation of excreta disposal and sanitation goals to 2030 for Costa Rica, were evaluated using data from the WHO and the National Water Laboratory (LNA). Results showed that, in the scenario where septic tanks are considered Safely Managed sanitation services, the country reaches 87,6\% of the population covered. While when they are not considered as Safely managed sanitation services, a 13\% coverage is achieved. Costa Rica reach the fifth position in coverage of basic hygiene between 13 countries of Latin-American. A strict monitoring of public policies related to sanitation is necessary in order to improve the country's indicators in the context of the SDGs.
\end{abstract}

\section{Introducción}

En el Objetivo 7 de los Objetivos de Desarrollo del Milenio (ODM) se propuso la meta de "Reducir a la mitad para el 2015, la proporción de personas sin acceso sostenible a agua potable y a servicios básicos de saneamiento."[1]. Con la intención de medir los avances alcanzados por cada país en estos temas, la OMS y la UNICEF crearon el Programa Conjunto de Monitoreo (PCM) [2]. EL PCM oficializó conceptos e indicadores de saneamiento para el monitoreo de las metas propuestas. 
Los conceptos de Instalaciones de saneamiento mejoradas e Instalaciones de saneamiento no mejoradas, incluyen mecanismos de medición como: disposición de excretas mediante alcantarillado con planta de tratamiento; el uso de alcantarillado sin tratamiento; uso de tanque sépticos; y el uso de letrinas sépticas [3].

Los datos de línea base de 189 países se establecieron con las coberturas del año 1990. Mientras que los avances alcanzados se oficializaron en el Informe titulado 25 Progresos en materia de Saneamiento y Agua Potable 2015 [4]; este mismo documento indica que, a nivel global, los países pasaron de 54\% de cobertura con Instalaciones de saneamiento mejoradas en 1990 a $68 \%$ en el 2015.

Con la publicación de los Objetivo de Desarrollo Sostenible (ODS), y específicamente en el Objetivo 6 (Agua Limpia y Saneamiento), se sustituyó el concepto de Instalaciones de saneamiento mejoradas por Servicios de saneamiento gestionados de forma segura, definidos como "Instalaciones privadas mejoradas donde los desechos fecales se depositan en un sitio de manera segura o se transportan y se tratan fuera del lugar, además de un lavado (lavatorio) de manos con agua y jabón" [5][6][7].

En Costa Rica el Laboratorio Nacional de Aguas (LNA) inició desde 1991 la preparación de informes anuales de cobertura y calidad del agua y saneamiento en Costa Rica [8][9][10]. Basándose en la premisa de que el acceso a agua potable, la adecuada disposición de excretas y la higiene en general, tienen una influencia directa sobre la prevención de enfermedades [11] [12][13] y fundamentados en el cambio de los ODM a los ODS se presenta este estudio, con el objetivo de abordar la variación de conceptos y datos de saneamiento, tomando como línea base los datos obtenidos en el periodo 2015, además de la formulación de metas país para el año 2030.

\section{Metodología}

Para efectos del presente artículo, se consideró necesario establecer dos escenarios para valorar los avances de Costa Rica en la disposición adecuada de excretas:

Escenario 1. En los Servicios de saneamientos gestionados de manera segura se tomaron en cuenta solamente las instalaciones privadas mejoradas, donde los desechos fecales se depositan en un sitio de manera segura o se transportan y se tratan fuera de lugar (que no incluye los tanques sépticos), además de un lavado de manos con agua y jabón.

Escenario 2. Se consideró el uso de tanque séptico más alcantarillado y otros tipos de tratamiento, además del lavado de manos con agua y jabón dentro de los Servicios de saneamientos gestionados de forma segura.

Coberturas con Instalaciones de saneamiento mejoradas en Costa Rica: 1990-2015

Con datos aportados por la OMS/UNICEF," se establecieron las Escaleras de Saneamiento para los años 1990 y 2015, basados en la evaluación realizada a los ODM por parte del PCM.

\section{Evaluación de disposición de excretas}

La evaluación de la disposición de excretas se llevó a cabo tanto a nivel nacional como internacional. El avance de cobertura de saneamiento a nivel nacional se estimó mediante los informes anuales del LNA de 1991 [14], 2015 [15] y la Encuesta Nacional de Hogares (ENAHO) [16] del Instituto Nacional de Estadística y Censos (INEC).

Los datos de los avances en la cobertura con Instalaciones de saneamiento mejoradas, usadas para la disposición de excretas en Costa Rica, se compararon a nivel de Latinoamérica, el Caribe y el resto del mundo, mediante el informe de OMS/UNICEF 25 Progresos en Materia de Saneamiento y Agua Potable, actualización al 2015 y evaluación de los ODM. 


\section{Aplicación de Escaleras de Saneamiento}

Utilizando los datos obtenidos en los informes antes mencionados, se aplicó la Escalera de Saneamiento de 1990 y 2015, esto dentro del marco de los ODM. Mientras que, utilizando el informe de Agua para Consumo Humano y Saneamiento en Costa Rica al Año 2017 en Viviendas y Más Allá del Hogar [17], se definió la Escalera de Saneamiento en el marcado de los ODS.

Proyectos de inversión en sistemas de tratamiento de aguas residuales en Costa Rica

Con fundamento en el Plan Nacional de Inversión en Saneamiento 2016-2045 [18], dentro del marco de la Política Nacional de Saneamiento en Aguas Residuales [19], se identificaron los proyectos de inversión a realizar por parte del Instituto Costarricense de Acueductos y Alcantarillados (AyA) en el periodo 2016 al 2045 en Costa Rica.

Metas propuestas para saneamiento al 2030

Los datos línea base del informe Agua para Consumo Humano y Saneamiento en Costa Rica al 2015. Metas al 2022 y 2030" [20], fueron utilizados para proponer una meta en saneamiento al año 2030.

\section{Situación de saneamiento e higiene en Costa Rica y América Latina}

Utilizando el documento "Progresos en Materia de Agua Potable, Saneamiento e Higiene 2017", se obtuvieron los resultados porcentuales de cobertura, aportados por OMS/JMP/UNICEF, y se elaboró la Escalera de la Higiene para Costa Rica. Además, se realizó la comparación con algunos países de América Latina.

\section{Resultados}

Coberturas de saneamiento en aguas residuales de Costa Rica 1990-2015 en el contexto latinoamericano y el mundo

En el cuadro 1 se presentan los datos de cobertura de disposición de excretas en Costa Rica, Latinoamérica y a nivel mundial.

Cuadro 1. Uso de Instalaciones de saneamiento mejoradas. Porcentaje de cobertura en Costa Rica, Latinoamérica y el mundo 1990-2015

\begin{tabular}{|c|c|c|c|c|c|}
\hline \multirow{2}{*}{ Región } & Período (tiempo) & Mejoradas & Compartidas & $\begin{array}{c}\text { Otras no } \\
\text { mejoradas }\end{array}$ & $\begin{array}{c}\text { Disposición a } \\
\text { cielo abierto }\end{array}$ \\
\hline \multirow{2}{*}{ Costa Rica } & 1990 & 88 & 4 & 6 & 2 \\
\cline { 2 - 6 } & 2015 & 95 & 4 & 1 & 11 \\
\hline \multirow{2}{*}{ Latinoamérica } & 1990 & 67 & 73 & 7 & 7 \\
\cline { 2 - 6 } & 2015 & 54 & 5 & 17 & 24 \\
\hline \multirow{2}{*}{ Mundo } & 1990 & 68 & 9 & 10 & 13 \\
\hline
\end{tabular}

Fuente: OMS/UNICEF 
Datos de saneamiento a nivel nacional

Con los datos del documento sobre la "Situación Actual de Agua de Consumo Humano y Aguas Residuales en Costa Rica 1991", con datos reales de 1990, y de la ENAHO a julio del 2015, se resumen, en el cuadro 2, los resultados de disposición de excretas en Costa Rica para ambos periodos.

Cuadro 2. Datos de disposición de excretas en Costa Rica en los años 1990 y 2015.

\begin{tabular}{|c|c|c|}
\hline Tipo de disposición & 1990 & 2015 \\
\hline Alcantarillado & $20,5 \%$ & $21,1 \%$ \\
\hline Tanques sépticos & $73,5 \%$ & $76,9 \%$ \\
\hline Letrinas & $3,0 \%$ & $1,6 \%$ \\
\hline Defecación a cielo abierto & $3,0 \%$ & $0,4 \%$ \\
\hline Total & $100 \%$ & $100 \%$ \\
\hline
\end{tabular}

Fuente: LNA

\section{Aplicación de las "Escaleras de Saneamiento" según conceptos} de los ODM en Costa Rica: 1990-2015

En las figura 1, se presentan las Escaleras de Saneamiento o disposición de excretas para los años 1990 y 2015, según los ODM; con la comparación de ambas figuras se observa el avance de Costa Rica en este tema, pasando de una cobertura nacional de $88 \%$ a 95\% con Instalaciones de saneamiento mejoradas entre ambos periodos.
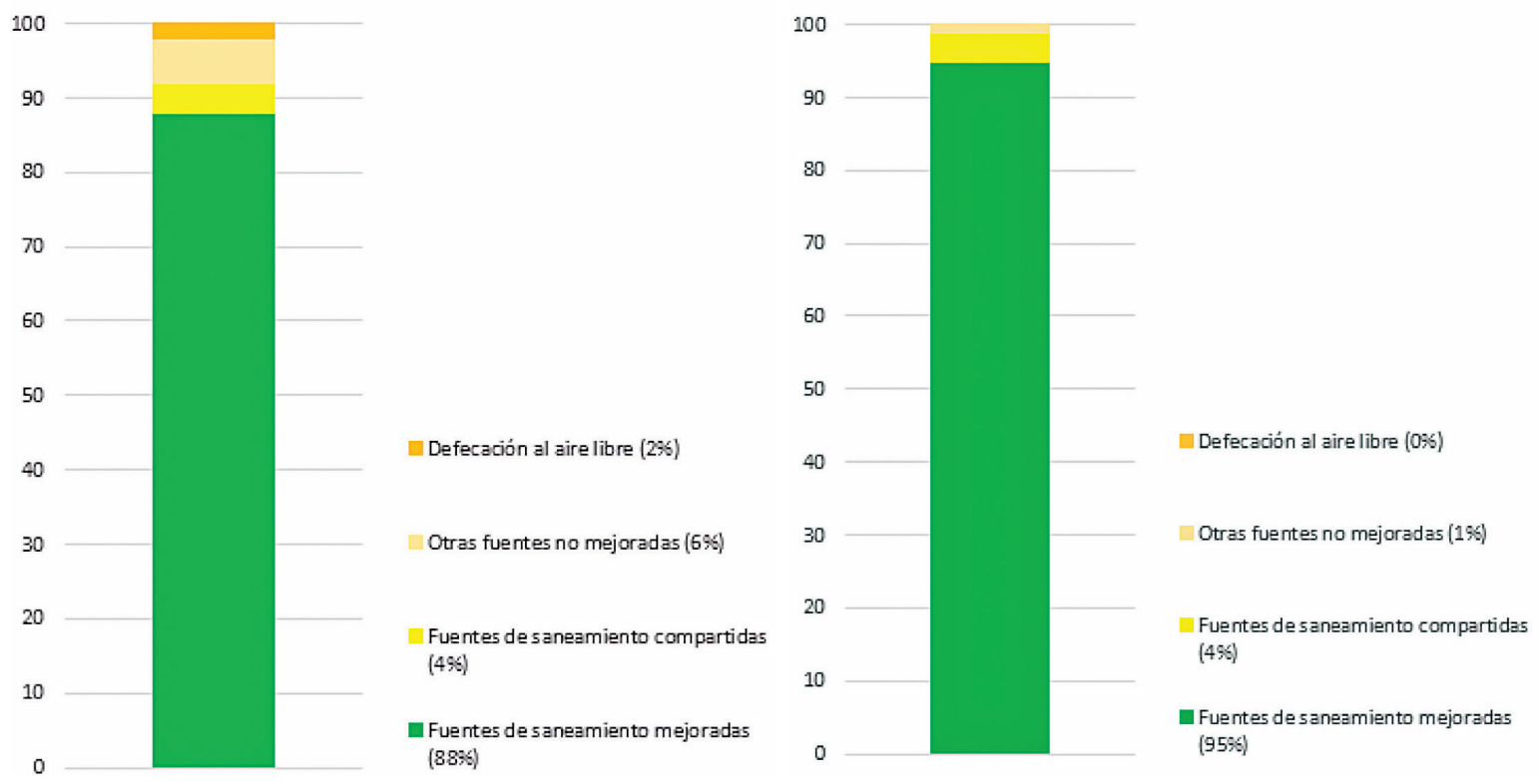

Figura 1. Escaleras de saneamiento según los ODM en 1990 (izq.) y 2015 (der.). Fuente: LNA 
Escalera de los Servicios de saneamiento gestionados de forma segura, de acuerdo con los escenarios 1 y 2.

En la figura 2, se presentan las Escaleras de los Servicios de Saneamiento gestionados en forma segura en Costa Rica para el año 2017.
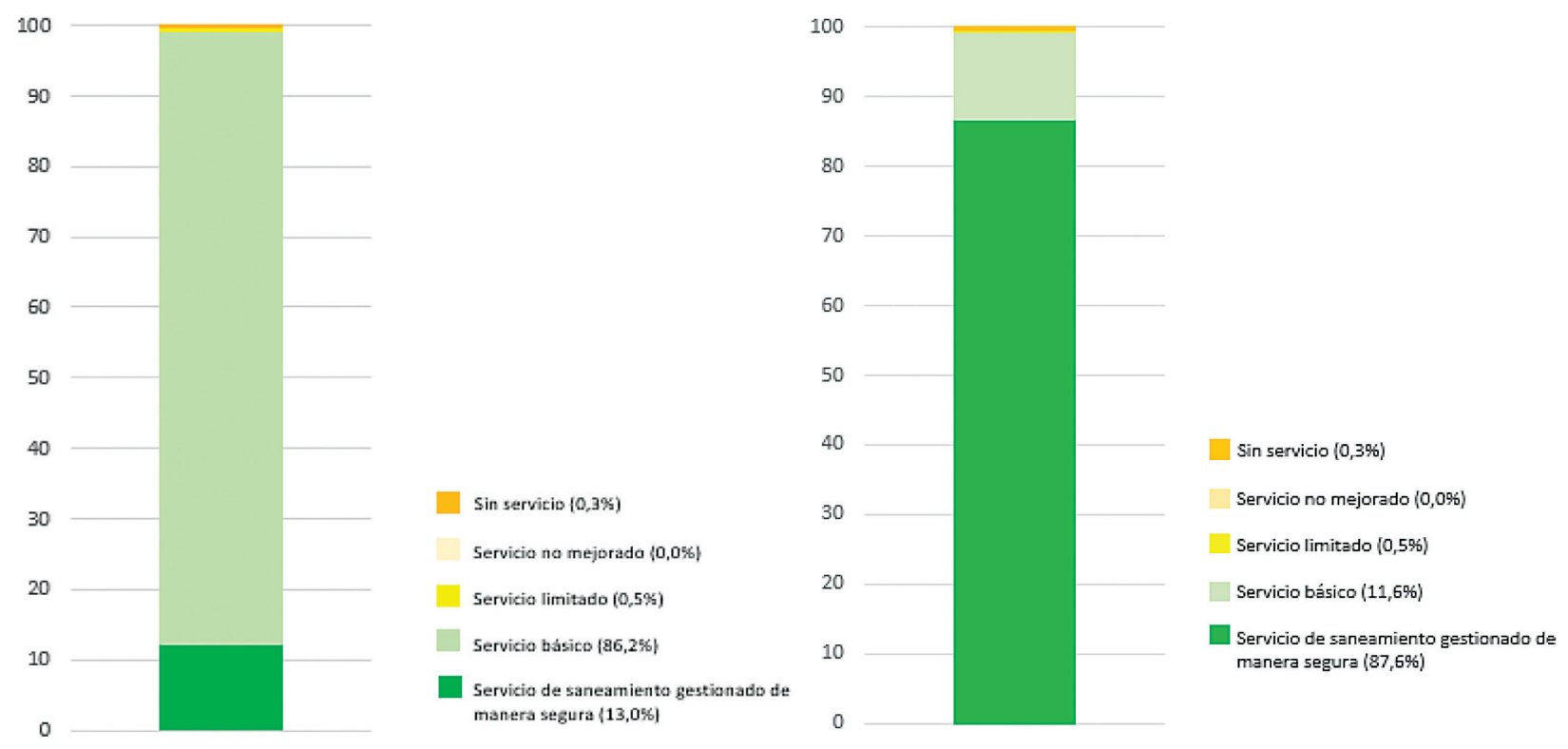

Figura 2. Escaleras de Saneamiento en Costa Rica según ODS para el año 2017 contemplando dos escenarios; Escenario 1 (izq) y Escenario 2 (der.). Fuente: LNA

Identificación de los proyectos de inversión en alcantarillado en Costa Rica: 2016-2045

De acuerdo con el AyA, los proyectos institucionales de inversión en alcantarillado para Costa Rica, entre los años 2010 y 2045, son los mostrados en el cuadro 3.

Cuadro 3. Beneficios de los Proyectos Utilizados para el Cálculo de los Costos Unitarios

\begin{tabular}{|c|c|c|}
\hline Proyecto-Ciudad & Conexiones & Proyección \\
\hline Palmares & 2.693 & Año 10 \\
\hline Jacó & 2.877 & Año 10 \\
\hline Nicoya & 6.272 & Año 12 \\
\hline Quepos & 6.096 & Año 12 \\
\hline Golfito & 2.374 & Año 12 \\
\hline Sardinal-El Coco & 5.880 & Año 10 \\
\hline Área Metropolitana Redes Sur & 7.028 & Dato único \\
\hline Área Metropolitana Redes Norte & 11.594 & Dato único \\
\hline
\end{tabular}

Fuente: Estudios de los proyectos y ofertas adjudicadas, AyA. 
Metas al 2030 en Servicios de saneamiento gestionados de forma segura

En la figura 3, se proyectan las metas al 2030, en cuanto al Servicio de saneamiento gestionado de forma segura, utilizando tanto el escenario 1 como el escenario 2 propuestos anteriormente.
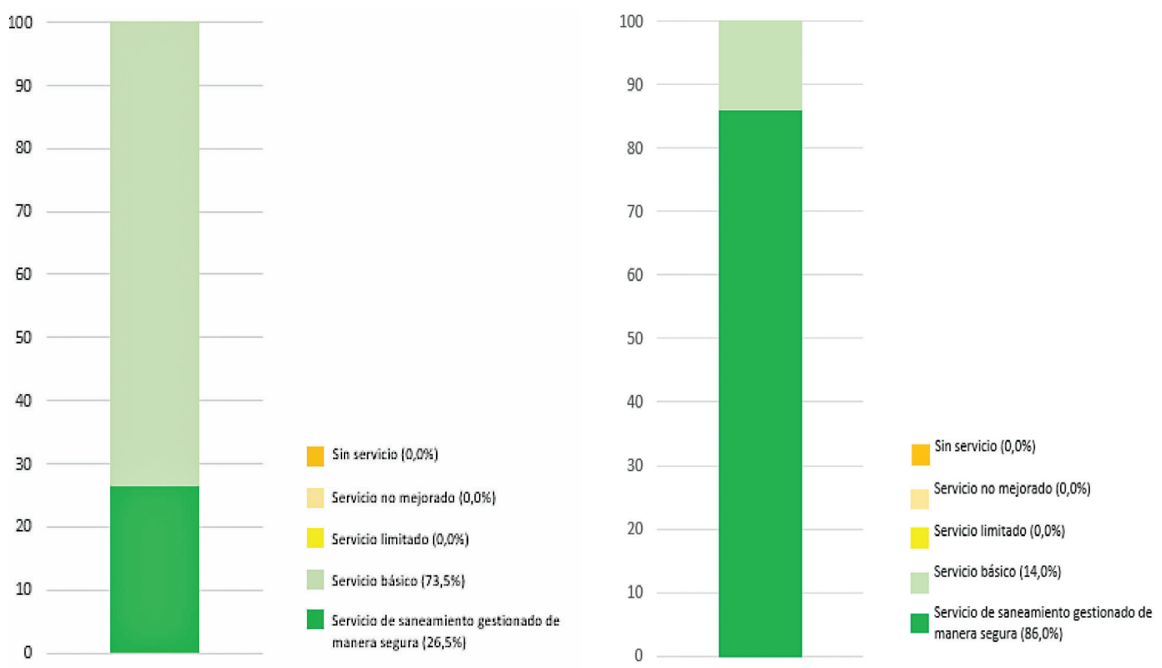

Figura 3. Escaleras de Saneamiento según los ODS en Costa Rica: proyección al año 2030 para el escenario 1 (izq.) y el escenario 2 (der.). Fuente: LNA.

Situación de la higiene en América Latina y Escalera de la higiene en Costa Rica

El cuadro 4 muestra los resultados porcentuales de cobertura obtenidos por 13 países de Latinoamérica y El Caribe en lo referente a la higiene, del año 2015, contemplada en el nuevo concepto de "Servicio de saneamiento gestionado de forma segura", según datos de OMS/ JMP/UNICEF.

Cuadro 4. Datos Porcentuales sobre Clasificación de la Higiene en América Latina y El Caribe 2015.

\begin{tabular}{|c|c|c|c|}
\hline País & Básico & Limitado & Sin instalación \\
\hline Belice & 87 & 8 & 5 \\
\hline Costa Rica & 84 & 10 & 6 \\
\hline Cuba & 85 & 10 & 5 \\
\hline Ecuador & 85 & 14 & 1 \\
\hline El Salvador & 90 & 7 & 3 \\
\hline Guatemala & 77 & 21 & 3 \\
\hline Guyana & 77 & 11 & 12 \\
\hline Haití & 26 & 42 & 6 \\
\hline Honduras & 84 & 10 & 17 \\
\hline Jamaica & 66 & 9 & 3 \\
\hline México & 88 & 16 & 29 \\
\hline República Dominicana & 55 & 8 & 5 \\
\hline Santa Lucía & 87 & & 6 \\
\hline
\end{tabular}

Fuente: OMS/JMP/UNICEF 


\section{Análisis de resultados}

\section{Evolución de los conceptos en saneamiento}

Con el cambio del ODM 7 al ODS 6, el PCM evolucionó del concepto de Instalaciones de saneamiento mejoradas al de Servicio de saneamiento gestionado de forma segura; este cambio radica, principalmente, en la inclusión del tratamiento de las aguas residuales al concepto de la variable. En el periodo de transición del 2015 al 2017, las diferentes naciones del mundo han tenido que establecer los datos línea base en cuanto al Servicio de saneamiento gestionado de forma segura; por esta razón, aún en el informe de Progresos en Materia de Agua Potable, Saneamiento e Higiene del 2017, se presentan datos de saneamiento básico utilizando el concepto de Instalaciones de saneamiento mejoradas en lugar de la cobertura con Servicio de saneamiento gestionado de forma segura.

Datos comparativos entre OMS/UNICEF de Instalaciones de saneamiento mejoradas en Costa Rica, Latinoamérica y el mundo

Los datos de OMS/UNICEF en Instalaciones de saneamiento mejoradas, indican que Costa Rica pasó de 88\% a 95\% de cobertura entre 1990 y el 2015. A su vez, el dato en Latinoamérica, en el mismo periodo, pasó de un $67 \%$ a $83 \%$ y a nivel mundial el avance fue de $54 \%$ a $68 \%$. De acuerdo con estudios del LNA, el avance a nivel nacional fue de 97\% en 1990 a 99,6\% en el 2015; no obstante, el nuevo concepto de Servicio de saneamiento gestionado de forma segura demuestra que los datos no han sido tan satisfactorios, como se observa en el desarrollo del presente estudio.

Escalera del Servicio de saneamiento gestionado de forma segura en Costa Rica al 2017 de acuerdo con los escenarios 1 y 2

En el escenario 1, o estricto, el "Servicio de saneamiento gestionado de forma segura" alcanzó el $13 \%$ en el 2017 , debido a que este dato no considera el $74,6 \%$ de cobertura de población que usaba el tanque séptico como medio de disposición de excretas; esto se debe a que los lodos de los mismos no se tratan en plantas de tratamiento. Con el escenario 2, el 74,6\% de cobertura con tanque séptico se suma al $13 \%$ de cobertura de alcantarillado con tratamiento, dando como resultado un $87,6 \%$ de población cubierta con "Servicio de saneamiento gestionado de forma segura".

Proyectos de inversión en sistemas de tratamiento de aguas residuales en Costa Rica

A través del Plan Nacional de Inversiones en Saneamiento 2016-2045, el AyA tiene planificado invertir recursos económicos en ocho nuevos proyectos de saneamiento, con la intención de recolectar y tratar las aguas residuales de diferentes sectores del país; los mismos estarán localizados en Palmares, Jacó, Nicoya, Quepos, Golfito, Sardinal-El Coco y el Área Metropolitana Etapas 1, 2 y las las Redes Norte y Sur. Estos nuevos proyectos abarcarán más de 45.000 conexiones, que beneficiarían a aproximadamente 969.615 personas de zonas urbanas y rurales, considerando un factor vivienda de 3,1 personas por conexión. Estos proyectos de inversión responden a la "Política Nacional de Saneamiento en Aguas Residuales" (PNSAR) para el periodo 2017-2045, propuesta por el Ministerio del Ambiente y Energía, el Ministerio de Salud y el AyA en el año 2016.

\section{Metas del Servicio de Saneamiento Gestionado de Manera Segura al 2030}

La Meta del ODS 6 indica que los países deben reducir en un 50\% la cantidad de aguas residuales sin tratamiento para el año 2030, tomando como línea base los datos del 2015. Datos del LNA indican que en el 2015 Costa Rica contaba con 9,22\% de población cubierta 
con aguas residuales tratadas, lo que permite concluir que el $90,78 \%$ de las aguas residuales no reciben tratamiento; este resultado se divide entre dos para obtener el $50 \%$, dando como resultado $45,39 \%$, que sumado al 9,22\% ya existente al 2015 nos da un valor meta de 54,6\% para el 2030.

Al igual que en el año 2017, se establecieron dos escenarios para estimar las metas de Costa Rica con Servicios de saneamiento gestionados de forma segura para el año 2030, según los ODS. En el caso del escenario 1, y bajo el supuesto de que los lodos generados por los tanques sépticos seguirán sin tratarse en plantas de tratamiento, por lo que estos últimos no calificarían para ser considerados como Servicios de saneamiento gestionados de forma segura, la cobertura alcanzará el 26,5\%, obtenido de sumar el 9,22\% de población cubierta con Servicios de saneamiento gestionados de forma segura existente en el año 2015, más el $17,43 \%$ de cobertura obtenida a través de los proyectos de inversión. Esta posibilidad sería una realidad siempre y cuando el país cumpla con la ejecución de los proyectos propuestos en el "Plan Nacional de Inversiones en Saneamiento 2017-2045".

Como se aprecia bajo este escenario, Costa Rica no lograría cumplir con la meta de 54,6\% de cobertura con Servicios de saneamiento gestionados de forma segura. En este escenario, se manifiesta la necesidad de tratar la totalidad de los lodos de los tanques sépticos a través de plantas de tratamiento regionales.

En el escenario 2, la cobertura con tanque séptico es considerada parte de un tratamiento de aguas residuales In situ. Al dato de cobertura con tanque séptico de 76,9\%, de la línea base 2015 , se le reduce el $17,43 \%$ por la sustitución de los tanques sépticos con los nuevos proyectos de inversión del Plan Nacional de Inversiones en Saneamiento 2017-2045, para contar con una cobertura de 59,47\% con tanque séptico en el año 2030; este resultado, sumado al 26,5\% de población cubierta con Servicios de saneamiento gestionados de forma segura, permitiría alcanzar una cobertura total de $86,0 \%$ a ese periodo, con lo cual el país estaría cumpliendo con la meta ODS.

\section{Escalera de la higiene aplicada en Costa Rica}

Los datos de OMS/JMP/UNICEF sobre higiene, indican que un 84\% de la población de Costa Rica tiene "Disponibilidad de una instalación de lavado de manos en la vivienda con jabón y agua" definida como Básico, $10 \%$ se clasifica como "Limitado" porque cuenta con "Disponibilidad de una instalación de lavado de manos en la vivienda sin jabón y agua", y 6\% se clasifica como "Sin instalación", debido a que "No existe instalación de lavado de manos en la vivienda".

\section{Conclusiones}

El cambio del concepto de Instalaciones de saneamiento mejoradas (ODM) a Saneamiento gestionado de forma segura (ODS), genera diferencias en los datos reportados durante la transición entre ambas iniciativas, con variaciones que podrían resultar muy significativas, como puede apreciarse al comparar los dos escenarios presentados para el año 2017 y las metas esperadas para el 2030.

La transición de los ODM a los ODS genera una falta de claridad a la hora de definir los conceptos incluidos en la Escalera de Saneamiento, especialmente al momento de clasificar la cobertura de saneamiento por tanques sépticos.

El hecho de no considerar los tanques sépticos como Estaciones de saneamiento mejoradas, imposibilita que Costa Rica cumpla con la meta país según los ODS, ya que no alcanzaría un $50 \%$ de saneamiento. 
Cuando se consideran los tanques sépticos como Estaciones de saneamiento mejoradas, se cumple la meta país de cobertura de saneamiento según los indicadores de los ODS (54,6\%).

Considerando la situación planteada en este artículo, la información anual reportada será insuficiente para poder dar respuesta completa a los indicadores de los ODS.

Costa Rica, en conjunto con Honduras, ocupan el quinto lugar en cobertura con servicio "Básico" de higiene entre 13 países latinoamericanos evaluados, por debajo de El Salvador (90\%), México (88\%), Belice y Santa Lucía (87\%), además de Cuba y Ecuador (85\%).

La Encuesta Nacional de Hogares (ENAHO) que se realiza en Costa Rica, no consulta sobre la presencia, dentro o fuera del servicio sanitario, de dispositivos para el lavado de manos con agua y jabón, como lo contemplan los ODS.

\section{Recomendaciones}

El cumplimiento del Plan Nacional de Inversiones en Saneamiento 2016-2045 por parte de AyA, representa un primer pero insuficiente paso para cumplir con las metas planteadas en el marco de los ODS.

La Política Nacional de Saneamiento en Aguas Residuales debe buscar mayor inversión a nivel de país, la cual durante muchos años se ha visto postergada con los consecuentes impactos ambientales, políticos, sociales y económicos que hoy día presenta nuestro país en esta materia.

Se debe aclarar, por parte de la OMS/UNICEF, si el tratamiento de los lodos de los tanques sépticos y el alcantarillado sanitario, están contemplados en el concepto Servicio de saneamiento gestionado de forma segura, con la intención de saber con cual escenario reportar en la clasificación de la Escalera de Saneamiento y el cumplimiento de las metas de los ODS.

EI INEC deberá realizar los ajustes necesarios en la Encuesta Nacional de Hogares, para poder obtener la información necesaria que permita dar una respuesta real a los indicadores solicitados, tanto en agua para consumo como en saneamiento e higiene.

\section{Referencias}

[1] Barboza, Karol; Ortega, Warren. Objetivos de Desarrollo del Milenio. III Informe de País 2015. San José, Costa Rica. MIDEPLAN; 2015; pag 1-99.

[2] Organización Mundial de la Salud. Seguimiento y Datos Empíricos Sobre Agua y Saneamiento. OMS. Ginebra, Suiza; Documento en línea en: https://www.who.int/water_sanitation_health/monitoring/es/

[3] Organización Mundial de la Salud y Fondo de las Naciones Unidas para la Infancia. Definición de Indicadores. OMS/UNICEF. Documento en línea en: www.who.int/water_sanitation_health/monitoring/evalamitad2.pdf

[4] Organización Mundial de la Salud, Fondo de las Naciones Unidas para la Infancia y Programa Conjunto de Monitoreo. 25 Progresos en Materia de Saneamiento y Agua Potable. Informe de Actualización 2015 y Evaluación de ODM/UNICEF OMS/UNICEF/PCM. Ginebra, Suiza; 2015:sp.

[5] Organización de las Naciones Unidas. Asamblea General de las Naciones Unidas. OMS/UNICEF. Documento en línea en: www.un.org/es/ga/about/

[6] Programa de las Naciones Unidas para el Desarrollo. Objetivo 6. Agua Limpia y Saneamiento. PNUD-Costa Rica. Documento en línea en: www.undp.org/content/undp/es/home/sustainable-development-goals/goal6-clean-water-and-sanitation.html

[7] Organización Mundial de la Salud, JMP y Fondo de las Naciones Unidas para la Infancia. Progresos en Materia de Agua Potable, Saneamiento e Higiene 2017. OMS/JMP/UNICEF; Nueva York. EUA; 2018 : PAG 1-39.

[8] Mora, Darner; Portuguez, Carlos. Situación de Cobertura y Calidad del Agua para Consumo Humano y Disposición de Excretas a Finales del 2001. Laboratorio Nacional de Aguas, Tres Ríos, La Unión, Cartago; 2002: sp.

[9] Mora, Darner; Portuguez, Carlos. Estado del Agua para Consumo Humano y Saneamiento al Año 2007. Laboratorio Nacional de Aguas, Tres Ríos, La Unión, Cartago. LNA; 2008: pag 1-27. 
[10] Mora, Darner; Mata, Ana; Portuguez, Carlos. Acceso a Agua para Consumo Humano y Saneamiento: Evolución en el Periodo 1990-2010 en Costa Rica Laboratorio Nacional de Aguas, Tres Ríos, La Unión, Cartago; 2011: pag 1-25.

[11] Heller, Leo. Saneamiento y Salud. Brasilia, Brasil. OPS/OMS/CEPIS; 1997: pp 1-83.

[12] Mora, Darner. Saneamiento, Educación y Salud. San José, Costa Rica. Editorama; 2005: 1-122.

[13] Organización Mundial de la Salud y Organización Panamericana de la Salud. La Salud y el Ambiente en el Desarrollo Sostenible. OMS/OPS; Washington D.C.; Publicación científica N572; 2000; sp.

[14] Mora, Darner. Situación Actual del Agua de Consumo Humano y las Aguas Residuales en Costa Rica 1991. San José, Costa Rica. Revista Biocenosis. Vol.2 enero-junio; 1991: pag 74-81.

[15] Mora, Darner; Mata, Ana; Portuguez, Carlos. Agua para Consumo Humano y Saneamiento y su Relación con los Indicadores Básicos de Salud en Costa Rica. Objetivos de Desarrollo del Milenio y la Agenda para el 2030. Laboratorio Nacional de Aguas, Tres Ríos, La Unión, Cartago; 2018: pag 1-28.

[16] Instituto Nacional de Estadística y Censos. Encuesta Nacional de Hogares 2017. INEC, San José, Costa Rica; Cuadros 5, 6 y 7 .

[17] Mora, Darner; Portuguez, Carlos. Agua para Consumo Humano y Saneamiento en Costa Rica al Año 2017 en Viviendas y Más Allá del Hogar. La Unión, Cartago. Laboratorio Nacional de Aguas; 2018: pag 1-28.

[18] Astorga, Yamileth y colaboradores. Plan Nacional de Inversiones en Saneamiento 2016-2045. AyA, BCIE, BID, KFW; San José, Costa Rica; 2017: pag 1-26.

[19] Instituto Costarricense de Acuedcutos y Alcantarillados. Politica Nacional de Saneamiento en Aguas Residuales: 2016-2045. AyA. San José, Costa Rica; 2016: pag 1-99.

[20] Mora, Darner; Portuguez, Carlos. Agua para Consumo Humano y Saneamiento en Costa Rica. Metas al 2022 y 2030. Laboratorio Nacional de Aguas; Tres Ríos, La Unión, Cartago; 2016. 